\title{
Ciencias de la implementación: de la evidencia a la práctica
}

\section{Implementation science: from evidence to practice}

\author{
Fernando Suárez-Obando, Carlos Gómez-Restrepo, Sergio Mario Castro-Díaz \\ - Bogotá, D.C. (Colombia)
}

\section{Resumen}

La amplia gama de evidencias científicas que respaldan diversas intervenciones clínicas no son suficientes para que dichas intervenciones se utilicen en la práctica. De tal modo que la Medicina Basada en la Evidencia debe dar el paso hacia la Practica Basada en la Evidencia, a través de procesos de implementación que permitan desplegar y contextualizar intervenciones en salud respaldadas por la evidencia. Para tal fin se ha desarrollado la Ciencia de la implementación, la cual investiga los factores que influencian el uso efectivo y completo de las innovaciones científicas en la práctica, intentando maximizar los beneficios de las intervenciones en salud.

Esta ciencia se define como el estudio de los métodos que promueven la incorporación sistemática de hallazgos de investigación en la rutina clínica, con el objetivo de mejorar la calidad y efectividad de los servicios e intervenciones en salud.

El propósito de este artículo es introducir el concepto de Ciencia de la Implementación en relación con la epidemiología clínica y establecer las razones por las cuales existe una imperiosa necesidad de su desarrollo.

Además, tiene el propósito de explicar el porqué de la necesidad de acelerar la aplicación efectiva de la Medicina Basada en Evidencia y definir los principales modelos que precisan el ámbito de trabajo de esta ciencia, incluyendo: las particularidades de los estudios de implementación, sus diferencias con los estudios clásicos de la epidemiología clínica, los modelos de implementación y evaluación, así como la definición de los desenlaces esperados en un estudio de implementación. (Acta Med Colomb 2018; 43: 207-216).

Palabras clave: ciencias de la implementación, medicina basada en la evidencia, práctica basada en la evidencia

\section{Abstract}

The wide range of scientific evidence supporting various clinical interventions is not sufficient for these interventions to be used in practice. For this reason, Evidence-Based Medicine must take the step towards Evidence-Based Practice, through implementation processes that allow deploying and contextualizing the health interventions supported by evidence. For this purpose, the Science of implementation has been developed, which investigates the factors that influence the effective and complete use of scientific innovations in practice, trying to maximize the benefits of health interventions.

This science is defined as the study of methods that promote the systematic incorporation of research findings in the clinical routine, with the aim of improving the quality and effectiveness of health services and interventions.

The purpose of this article is to introduce the concept of Implementation Science in relation to clinical epidemiology and establish the reasons by which there is an urgent need for its development.

In addition, it has the purpose of explaining why the need to accelerate the effective application of Evidence-Based Medicine and define the main models that define the scope of work of this science, including: the particularities of implementation studies, their differences with the classic studies of clinical epidemiology, the implementation and evaluation models, as well as the definition of expected outcomes in an implementation study. (Acta Med Colomb 2018; 43: 207-216).

Key words: implementation science, evidence-based medicine, evidence-based practice.
Dr. Fernando Suárez-Obando: Instituto de Genética Humana. Doctorado de Epidemiología Clínica, Departamento de Epidemiología y Bioestadística. Facultad de Medicina, Pontificia Universidad Javeriana, Hospital Universitario San Ignacio; Dr. Carlos Gómez-Restrepo: Doctorado de Epidemiología Clínica, Departamento de Epidemiología y Bioestadística, Facultad de Medicina. Pontificia Universidad Javeriana. Hospital Universitario San Ignacio; Dr. Sergio Castro: Doctorado de Epidemiología Clínica, Departamento de Epidemiología y Bioestadística, Facultad de Medicina, Pontificia Universidad Javeriana. Bogotá, D.C. (Colombia). Correspondencia: Dr. Fernando Suárez Obando, Bogotá, D.C. (Colombia).

This work was partially supported by grant \#U19MH109988 from the U.S. National Institute of Mental Health.

E-mail: fernando.suarez@javeriana.edu.co Recibido: 29/VI/2017 Aceptado: 8/XI/2017 


\section{Introducción}

La investigación en salud, desarrollada a través del modelo de la epidemiología clínica, tiene un elevado valor para la sociedad, dado que provee información significativa sobre las enfermedades, los factores de riesgo para padecerlas, los desenlaces clínicos de los tratamientos, los patrones de cuidado, el pronóstico y los costos asociados a la atención en salud $(1,2)$.

La investigación clínica genera evidencia que tiene como propósito ser utilizada en la toma de decisiones en la práctica clínica. El uso y confianza que se tiene acerca de dicha evidencia, depende tanto de la pregunta clínica, que el médico desea responder en su quehacer diario, como del diseño epidemiológico que se haya reportado para resolver dicha pregunta y del rigor científico con que se haya desarrollado el estudio $(3,4)$. Es así como, los experimentos clínicos proveen información sobre eficacia y seguridad de las intervenciones (farmacológicas o no), al controlar las variables que pueden impactar los resultados del experimento (3). Mientras que otros diseños, como las cohortes o los casos y controles, se orientan, por ejemplo, hacia la determinación de factores pronósticos y de riesgo (5).

El amplio espectro de acción de la investigación clínica, se ha extendido hasta la recopilación y análisis ordenado de la evidencia existente, generando nuevas metodologías de estudio de la evidencia, que incluyen las revisiones sistemáticas y los metaanálisis y ha desarrollado la recopilación experta de la literatura biomédica, bajo la presentación de las guías de práctica clínica, las cuales condensan la evidencia en acciones pragmáticas para el clínico (6), un modelo de desarrollo enmarcado en la corriente de la MBE (medicina basada en la evidencia) (7), el cual ha demostrado ser un modelo exitoso, en la generación de una amplia gama de evidencias, difundidas a través de literatura científica, en todas las áreas de la atención en salud (8).

Sin embargo, a pesar del creciente volumen de información y conocimiento generado por la investigación clínica, llevarlo a la práctica clínica de forma efectiva, es un aspecto que la propia investigación clínica no resuelve, dado que su objetivo principal, es generar conocimiento bajo las circunstancias propias del diseño epidemiológico, mas no necesariamente transferir el conocimiento de los resultados a entornos de práctica real (9), por tanto, uno de los retos más grandes que enfrentan actualmente los sistemas de salud, es dilucidar el mejor método para identificar intervenciones con eficacia demostrada y aplicarlas en la práctica real (10). A lo cual se suma, la necesidad de desarrollar investigaciones que sustenten los procesos decisorios en salud y la exigencia de ejecutar investigaciones promovidas por la demanda de servicios (11), es decir, establecer los factores contextuales que influyan en los efectos y resultados de las intervenciones.

La trasferencia de resultados de investigación, debe ser el paso obligatorio luego de concluir el proceso investigativo, sin embargo, este proceso ha demostrado ser irregular e incluso inexistente, es decir, que, a pesar del gran volumen de información, resultado de múltiples investigaciones, éste no ha sido llevado a la práctica y se constituye en sí mismo en una barrera entre la pretendida objetividad de la actuación científica y su propósito ético de resolver problemas reales. Es así como, coexisten intervenciones y conocimientos potencial o probadamente beneficiosos que no se utilizan en la práctica, mientras persisten acciones rutinarias que no tienen respaldo en la evidencia científica (12). De tal modo que, se requiere de un enfoque que permita superar las barreras entre la investigación, tanto clínica como básica y su aplicación en la atención de pacientes (13).

Con el propósito de resolver la brecha entre la producción del conocimiento respaldado por la evidencia científica y su posterior aplicación y uso en la práctica en escenarios reales, se ha desarrollado la denominada Ciencia de la Implementación (Implementation Science) la cual investiga los factores que influencian el uso efectivo y completo de las innovaciones científicas en la práctica, pretendiendo maximizar los efectos beneficiosos de las intervenciones en salud y que se define formalmente como el estudio de los métodos que promueven la incorporación sistemática de hallazgos de investigación en la rutina clínica, con el objetivo de mejorar la calidad y efectividad de los servicios de salud (14), contribuyendo así a maximizar los efectos favorables de las intervenciones de salud (15). Se trata de un campo relativamente nuevo dentro del sector salud y para el cual, la metodología de investigación sobre la implementación, es un asunto cuya magnitud es desconocida para una gran proporción de trabajadores e incluso, entre investigadores, del área de la salud (16). El propósito de este artículo es introducir el concepto de Ciencia de la Implementación en relación con la epidemiología clínica y establecer las razones por las cuales existe una imperiosa necesidad de su desarrollo, con el propósito de acelerar la aplicación efectiva de la MBE y definir los principales modelos que definen el ámbito de trabajo de esta ciencia.

\section{Particularidades de los estudios de implementación}

Existen diferencias fundamentales entre la investigación clínica y la investigación de implementación. En primer lugar, la unidad de análisis de la investigación clínica (específicamente en los experimentos clínicos) es propiamente el conjunto de desenlaces de interés relacionados con la intervención, el cual a su vez define la unidad de aleatorización y las medidas de desenlace (clínicas o no clínicas), como aquellas dimensiones que evalúan la intervención en estudio (17). Es así como los estudios de eficacia se fijan principalmente en la validez interna, estableciendo los límites de la inferencia causal entre el tratamiento o intervención y la evaluación de desenlaces, en otras palabras, los resultados de un experimento clínico se aplican a sujetos que en la práctica clínica, cumplieran los criterios de inclusión especificados en el estudio (18).

En contraste, en los diseños de investigación orientados a la efectividad clínica, en donde se evalúan costos o calidad 
de vida, la investigación se orienta principalmente hacia la validez externa (19). Estos estudios también se han definido como experimentos clínicos pragmáticos, que son aquellos en los cuales, la hipótesis y el diseño del estudio son desarrollados específicamente para responder a las preguntas de los tomadores de decisiones, caso en el cual se seleccionan intervenciones clínicamente relevantes para ser comparadas, se incluye población y sitios de práctica heterogéneos en los cuales se aplican las intervenciones y se recolectan amplios rangos de desenlaces en salud (20).

A diferencia de los estudios previamente mencionados, la investigación orientada a la implementación, se ocupa de estudiar la adopción, por parte de proveedores y sistemas de salud, de intervenciones clínicas respaldados por evidencia científica, pretendiendo llevar la MBE a la PBE (Prácticas Basadas en la Evidencia) (21). Como su objetivo es el estudio de estos determinantes que afectan el desempeño óptimo de las PBE, los desenlaces son los comportamientos del tipo: niveles o tasas de adopción y adherencia a la intervención, de tal modo que se asume la efectividad de la intervención y ésta no se incluye necesariamente, para su medición en el diseño, mientras que sí se resalta la caracterización del contexto en donde se implantará la intervención (22).

Se considera además que, la implementación abarca el despliegue y puesta en marcha de una gran variedad de intervenciones tales como políticas, programas, prácticas y servicios orientados a mejorar la salud de las personas (23). Casos en los cuales, de forma independiente al mecanismo a través del cual funcionen esas intervenciones, (ej.; prevención de enfermedades, promoción de la salud), la investigación sobre su implementación es fundamental para entender cómo se comportan el mundo real (24); pues en éste, los desenlaces de salud no están determinados sólo por las características de las intervenciones ya que también se afectan por el sistema de salud, políticas y situaciones específicas en los ámbitos psicosocial, económico y ambiental (25).

De estos aspectos se desprende que la evaluación contempla diversas perspectivas sobre la intervención a introducir, el significado de la intervención en el ámbito asistencial y cómo impacta en la práctica rutinaria, por tanto, los procesos de implementación, involucran agentes de diversas disciplinas que incluyen investigadores de servicios de salud, economistas, sociólogos, antropólogos, administradores, personal clínico y pacientes, los cuales son participantes de la investigación de la implementación, pero que usualmente no están involucrados en los ensayos clínicos controlados $(26,27)$. La investigación sobre implementación, demanda la participación de diversos interesados y se apoya en múltiples disciplinas con el propósito enfrentar a los desafíos de la implementación (28).

Cabe recalcar que, tanto en los procesos, como en la investigación sobre implementación, se destaca el rol de quienes trabajan directamente en la rutina de atención diaria, dado que estas personas están al corriente de los procesos, barreras y oportunidades en un sistema de salud o un nivel de atención primaria, de tal modo que pueden plantear los cuestionamientos adecuados que enriquecen y contextualizan el proceso de despliegue de un programa o una política (29).

Los estudios de Mejoramiento de la Calidad (Quality Improvement) tienen aspectos en común con los estudios de implementación, dado que comparten el propósito de mejorar la calidad de la atención en salud en contexto de práctica diaria, sin embargo, los estudios de mejoramiento en la calidad, parten de un problema específico que ha sido reconocido, ya sea a nivel del proveedor, del centro de atención clínico o del propio sistema de salud y que conlleva al diseño de estrategias que mejoren el problema específico y, como consecuencia se mejore el sistema de salud (30).

En el caso de la implementación, se aprovecha una estrategia, que ha sido probada bajo los parámetros de la MBE y que a su vez pretender solventar una carencia del proveedor, del centro de atención clínica o del sistema de salud y que tiene la pretensión de ser aplicada o generalizable más allá del sitio individual bajo estudio (31). La implementación permite que la MBE se ejecute y se manifieste en la vida real como una PBE.

En contraste con la implementación, la diseminación se refiere a la difusión de información acerca los resultados de la evaluación de una intervención clínica, de guías de práctica clínica o de la evaluación de impacto de una estrategia o política $(32,33)$. La diseminación puede estar asistida por diseños pedagógicos, tecnologías de la información y la comunicación $(34,35)$ y además, puede ser evaluada incluso con diseños de antes después (36). Si bien es cierto que la implementación incluye la diseminación de la información, ésta está incluida en un esfuerzo de mayor magnitud que pretende hacer efectiva una estrategia que debe ser informada adecuadamente a los interesados. La implementación impulsa la diseminación de la MBE con el propósito de estructurar la PBE. Este aspecto ha sido desarrollado de manera específica para la implementación de GPC (Guías de práctica clínica) en Colombia, en donde se hace una distinción clara entre de diseminación y difusión. La difusión hace referencia a procesos de distribución de información con el objetivo de presentarle a la sociedad, los grupos de interés y a los potenciales usuarios, las GPC, mientras que la diseminación indica los procesos comunicación efectiva y educación que tienen por objetivo mejorar el conocimiento y habilidades de los usuarios finales de las GPC, sean éstos prestadores de servicio o pacientes (37).

Mientras que la distinción entre la implementación y los demás tipos de estudios puede ser clara, vale la pena recalcar que en la práctica estas diferencias deben ser definidas desde el diseño del estudio, de tal modo que la generación de hipótesis y la metodología empleada, sea la adecuada para la medición de los desenlaces de interés (38).

Por ejemplo, si se estudia el efecto de un programa basado en TIC (Tecnologías de la Información y la Comunicación) que incrementa la efectividad de una intervención mixta farmacológica, nutricional y de terapia comportamental, 
en el manejo de la obesidad en adultos jóvenes, el impacto de la intervención, en el estado de salud de los sujetos, será una evaluación de una práctica basada en la evidencia, mientras que la medida de la proporción de médicos que utilizan como estrategia de manejo, la estrategia TIC y la intervención mixta, será una medida de un estudio de implementación (Tabla 1).

Sin embargo, a pesar de estas definiciones es difícil delimitar los límites de un estudio de implementación en relación con el ensayo pragmático, de tal modo que se ha planteado que un diseño de implementación es, en teoría, un ensayo pragmático, dado que este tipo de ensayo proporciona pruebas científicas y evidencia de la eficacia de la intervención o estrategia en condiciones de mundo real, en otras palabras, el enfoque de ensayo pragmático es útil para la implementación, la cual en si misma añade otros valores fundamentales sobre el proceso de despliegue de la intervención y sus consecuencias, las cuales van más allá de la eficacia.

\section{Estudios híbridos de implementación y efectividad}

Si bien es cierto que la implementación puede demostrar mejoría en la incorporación de PBE, el impacto de estas prácticas en los desenlaces de salud no es su principal objetivo (17), de tal modo que no se puede asumir que una implementación adecuada significa mejora en desenlaces clínicos. Así mismo, se deben conocer las razones que expliquen cómo los contextos y condiciones locales influyen en la efectividad. Por tanto, se ha establecido que existe la necesidad de integrar ambas aproximaciones, en los denominados estudios mixtos o híbridos, de efectividad e implementación (39).

Alrededor de este tema sean propuesto al menos tres tipos de estudios mixtos:
1. Diseño mixto tipo I, en el cual se evalúa, mediante desenlaces clínicos, el impacto en salud de la intervención, mientras se recopila información sobre la implementación.

2. Diseño mixto tipo II, en el cual se evalúa mediante desenlaces clínicos, el impacto en salud de la intervención y se evalúa la estrategia de implementación de acuerdo con su capacidad de incrementar el uso de PBE.

3. Diseño mixto tipo III, en el cual se evalúa la capacidad de la estrategia de implementación, para incrementar el uso de PBE y se recolecta información sobre el impacto en salud de la intervención (21).

El concepto de estudios híbridos refleja la continuidad existente entre diversos tipos de investigación y denota las dificultades que existen al tratar de definir una taxonomía absoluta de los tipos diseño y en definir sus límites.

De hecho, la noción de Medicina Traslacional implica que la investigación biomédica es un proceso continuo que parte desde la investigación básica, hasta su implementación y evaluación de impacto en la salud de las personas en contextos de práctica cotidiana (40). De tal modo que la implementación debe su pertinencia a ciertos momentos específicos de la investigación. Dentro de este proceso, los estudios de implementación se relacionarían con los desenlaces de salud poblacional a través de los estudios híbridos y de manera indirecta a través de informar el proceso de desarrollo de políticas que favorezcan y fortalezcan el uso de las PBE. En la Figura 1 se aprecia el proceso de investigación biomédica y el momento en que la implementación se hace pertinente y a su vez el instante en que se hace el principal objetivo de la investigación.

Además, los resultados de los estudios de implementación se han utilizado como material guía en la definición de las prioridades de patrocinio por parte de las diferentes agencias científicas (41). En la actualidad las prioridades

Tabla 1. Principales diferencias entre estudios epidemiológicos comparados con los estudios de implementación. Los estudios de implementación comprenden diversas características de los experimentos clínicos clásicos y de los estudios pragmáticos, además asume en su metodología aspectos y características de los estudios de calidad y diseminación.

\begin{tabular}{|c|c|c|c|c|c|}
\hline \multirow{2}{*}{$\begin{array}{l}\text { Características } \\
\text { del diseño }\end{array}$} & \multicolumn{5}{|c|}{ Estudio } \\
\hline & Experimento clínico & $\begin{array}{c}\text { Experimento } \\
\text { pragmático }\end{array}$ & $\begin{array}{c}\text { Estudio de } \\
\text { implementación }\end{array}$ & $\begin{array}{c}\text { Mejoramiento de } \\
\text { calidad }\end{array}$ & Diseminación \\
\hline Pregunta de investigación & $\begin{array}{l}\text { ¿Cuál intervención es } \\
\text { más eficaz y segura? }\end{array}$ & $\begin{array}{l}\text { ¿Cuál intervención es } \\
\text { más eficaz y segura? }\end{array}$ & $\begin{array}{l}\text { ¿Cuál es la magnitud } \\
\text { de adopción de la } \\
\text { intervención? }\end{array}$ & $\begin{array}{l}\text { ¿Qué tanto mejoró } \\
\text { la calidad de la } \\
\text { intervención luego del } \\
\text { proceso de mejora? }\end{array}$ & $\begin{array}{c}\text { ¿Cuál fue el grado } \\
\text { de apropiación de la } \\
\text { información sobre la } \\
\text { intervención en un grupo } \\
\text { u organización? * }\end{array}$ \\
\hline Parámetros** & $\mathrm{CI} / \mathrm{CE} \dagger$ & $\mathrm{CI} / \mathrm{CE}$ & Organización o sistema & Organización o sistema & Organización o sistema \\
\hline Unidad de estudio & Sujeto & Sujeto & $\begin{array}{c}\text { Sujeto/organización } \\
\text { /sistema } \neq\end{array}$ & $\begin{array}{c}\text { Sujeto/organización } \\
\text { /sistema }\end{array}$ & $\begin{array}{c}\text { Sujeto/organización } \\
\text { /sistema }\end{array}$ \\
\hline Asignación & Aleatorizada & Aleatorizada & $\begin{array}{c}\text { Aleatorizada / } \\
\text { no aleatorizada }\end{array}$ & No aleatoria & No aleatoria \\
\hline Brazos & Experimentales & Experimentales & Institución o servicio & Institución o servicio & Institución o servicio \\
\hline Contexto & Controlado & Vida real & Vida real & Vida real & Vida real \\
\hline Desenlaces esperados & Clínicos y seguridad & Clínicos y seguridad & $\begin{array}{c}\text { De aceptabilidad a } \\
\text { sostenibilidad }\end{array}$ & Incremento en la calidad & $\begin{array}{l}\text { Incremento en el } \\
\text { conocimiento }\end{array}$ \\
\hline Definición & Eficacia & Efectividad & Adopción & Optimización & Difusión \\
\hline
\end{tabular}


de investigación se definen a partir de resultados de investigaciones principalmente experimentales o algunas pesquisas de tipo poblacional (ej. encuestas de salud). Con la inclusión de las ciencias de implementación dentro del panorama de investigación y desarrollo de políticas de salud se generan datos que permitirían establecer prioridades en los otros momentos dentro del proceso de investigación médica. De esta manera podríamos decir que los estudios de implementación se encuentran directamente involucrados en dos momentos importantes del ciclo de investigación dentro de la noción de Medicina Traslacional: El paso a la práctica y el paso a las poblaciones. En la Figura 2 se muestra como se relaciona la ciencia de implementación con el proceso de investigación.

\section{Tipos de evaluación del proceso de implementación}

Teniendo en cuenta que las mediciones hechas en este tipo de estudios, se basan en la evaluación del transcurso de la implementación y en su impacto en el desarrollo y adopción de PBE, se hace necesario determinar los tipos de evaluación que se llevarán a cabo en el proceso, con el fin de determinar el tipo de datos que se deben evaluar.
En primer lugar y de manera general, se encuentra la Evaluación del Proceso, la cual describe las características de la implementación de la intervención, en el contexto real del sitio de despliegue (42). En este proceso, los datos son recolectados antes, durante y después de la implementación y analizados sin que se dé retroalimentación al equipo de implementación, de tal forma que no se interfiera o se interrumpa el proceso (17).

Esta evaluación puede iniciarse a través de un estudio observacional previo, que se desarrolle para la preparar la estrategia de implementación o durante el curso de cambios, planeados o no, de una política o un programa de atención para una enfermedad especifica. Por ejemplo, si se desea implementar una estrategia basada en la evidencia, en el mencionado programa apoyado por TIC, se puede caracterizar el uso previo de la estrategia o estrategias similares por parte del equipo médico y medir el uso del programa específico, junto a sus desenlaces, durante y después de la implementación, escenario en el cual el investigador actúa como observador pasivo mientras transcurre la implementación y su evaluación.

La medición de la implementación, a través de la evaluación del proceso se basa en valorar:

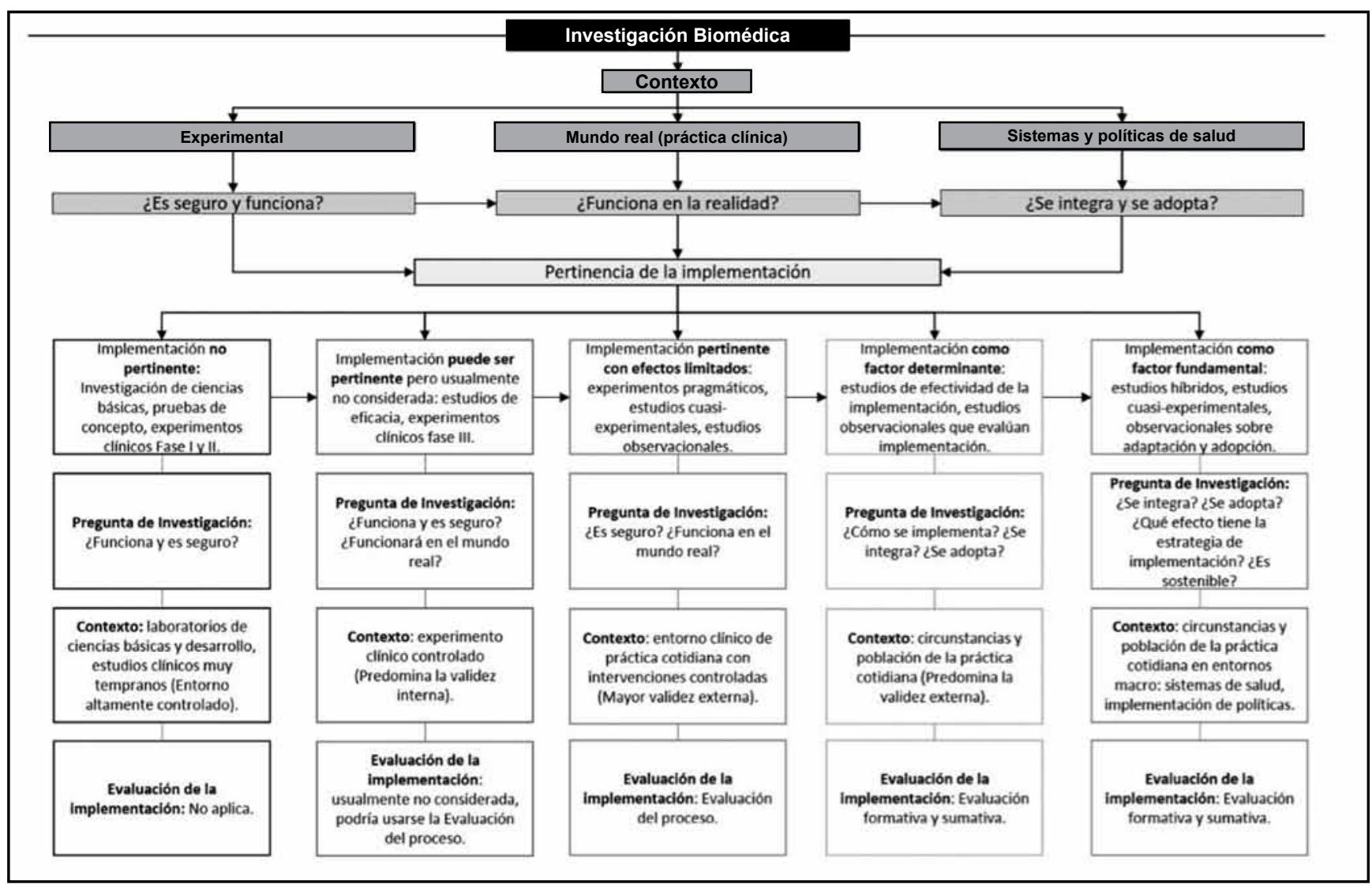

Figura 1. Investigación biomédica e implementación. La investigación biomédica, abarca diversos procesos que, a través de etapas sucesivas, pretenden llevar a la práctica la innovación y desarrollo. Los productos de las etapas experimentales y de innovación, se llevan a la práctica clínica, a través de investigaciones que implican diversas fases, cada una de las cuales es susceptible de procesos de implementación. A medida que la investigación establece la eficacia y la seguridad de las intervenciones, la posibilidad de implementación poco a poco se impone. Las preguntas de investigación y métodos de evaluación se superponen a lo largo de las diversas etapas, hasta alcanzar los procesos de implementación de intervenciones soportadas con evidencia científica, a lo largo de sistemas de salud, momento en el cual la integración, adopción y sostenibilidad cobran la mayor relevancia dentro del proceso. Adaptado de Peters y cols. [43]. 
1. El proceso de adopción del programa, es decir, cómo se desarrolló el reclutamiento de los centros, incluyendo los métodos de diseminación de la información,

2. La implementación propiamente dicha, estimando la fidelidad al programa, la cual surge de comparar el planteamiento original de intervenciones efectivas y las intervenciones finalmente implementadas.

La implementación, también se evalúa a través de la cobertura o alcance, la cual establece el número de personas (médicos y pacientes) que fueron alcanzados por el programa, esta medida puede ser complementada con el número de médicos que prescriben o ejecutan el programa y el número efectivo de sujetos que reciben el manejo por parte del programa. Además, la implementación también se evalúa con el grado de satisfacción, que los sujetos alcanzados, tuvieron con el programa.

3. La sostenibilidad o institucionalización de la intervención, es decir, la extensión a la cual el programa se incorpora en las rutinas diarias de los médicos en su organización y

4. El contexto o estudio de los determinantes del entorno que afectan la implementación del programa $(44,45)$. Estas medidas se incluyen dentro de los desenlaces esperados por la implementación (Tabla 1).

Además de la Evaluación del Proceso de implementación, se puede desarrollar otro tipo de evaluación, denominado Evaluación Formativa, que utiliza los mismos métodos que la evaluación del proceso, pero la información en este caso, sí es comunicada durante el desarrollo del estudio, al equipo de implementación y a los sujetos participantes objeto de la implementación, con el propósito de adaptar y mejorar el proceso de despliegue.

Es una evaluación que se debe definir a priori en la generación de la pregunta de investigación y el diseño del estudio $(17,46)$. Finalmente, en la medición del proceso de implementación, se puede utilizar un modelo de Evaluación Sumativa o de compilación, en el cual se evalúa, a través de desenlaces que pueden ser clínicos, el impacto de la implementación, fijándose además en cuantificar la magnitud de cambio en el uso de la estrategia implementada y la calidad de su uso luego de la implementación (47).

Tanto la Evaluación del Proceso, como la Evaluación Formativa se centran en el proceso, mientras que la Evaluación Sumativa se centra en el desenlace. Es en la Evaluación Sumativa en donde es frecuente encontrar el componente económico que estima el efecto en costos de la adopción de una política gubernamental o programa, en un sitio de atención clínica o en un sistema de salud completo (48).

Los resultados o desenlaces a partir de los cuales se determina el éxito o no de la implementación se describen en la Tabla 2. Cada tipo de evaluación cobra mayor relevancia dependiendo del momento de la investigación en el cual la implementación se lleve a cabo (Figura 1).

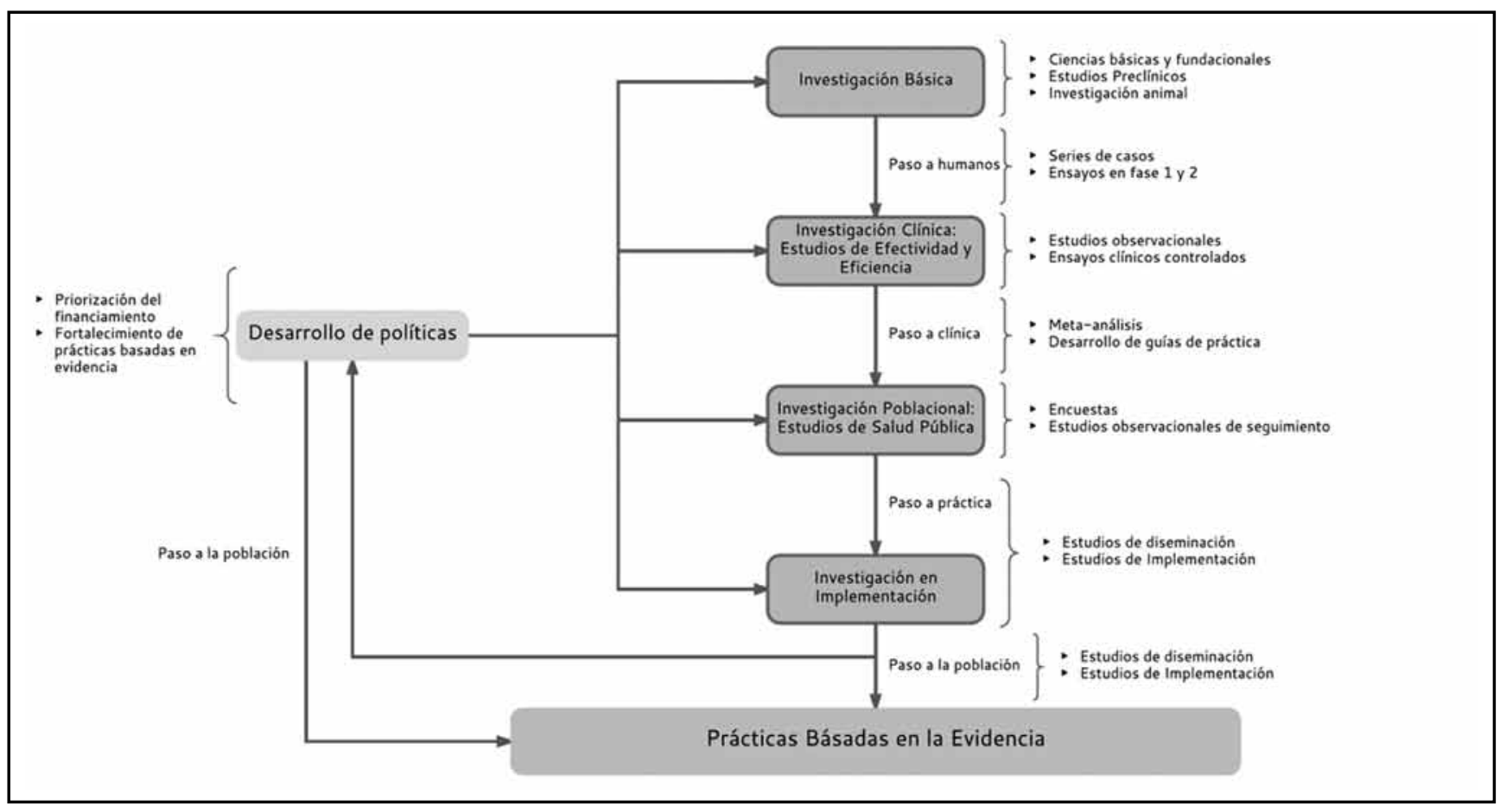

Figura 2. Implementación en el proceso de Medicina Traslacional. Los estudios de implementación se encuentran en la etapa final del proceso de investigación biomédica. Idealmente, se espera que los hallazgos biomédicos efectivos se integren dentro de las políticas y los sistemas de salud. Le compete a las ciencias de implementación enlazar los hallazgos científicos con la práctica clínica y con las agencias interesadas que desarrollan políticas y perfeccionan el sistema de salud. Por lo tanto tiene una relación directa con el eslabón final de la cadena de Medicina Traslacional y de manera indirecta alimenta el inicio de la cadena de investigación biomédica y ayuda a promover cambios que establecen las bases para que se generen otros desarrollos como la Medicina de Precisión. 


\section{Fuente de los datos y definición de desenlaces}

Los datos para llevar a cabo la evaluación de la implementación (sea la evaluación del proceso, evaluación formativa o sumativa) pueden provenir de diversas fuentes, de orden cualitativo o cuantitativo y que se valoran en diversos niveles de observación, que incluyen médicos, pacientes, usuarios de un servicio o programa, proveedores y personal administrativo (17).

Las medidas cuantitativas usuales incluyen entrevistas estructuradas, escalas y herramientas que evalúen el contexto organizacional y el flujo de trabajo (49) y además definen

Tabla 2. Definición de los resultados (desenlaces) de la implementación. El proceso de implementación requiere de desenlaces o resultados que den cuenta del éxito de la implementación, estos desenlaces pueden combinarse con desenlaces clínicos o con preguntas económicas en los estudios híbridos, desenlaces que pueden ser evaluados tanto en el proceso de implementación como a través de evaluación formativa o sumativa. Adaptado de Proctor y cols. (56) y Peters y cols. (43).

\begin{tabular}{|c|c|}
\hline $\begin{array}{l}\text { Desenlaces de la } \\
\text { implementación }\end{array}$ & Definición \\
\hline Aceptabilidad & $\begin{array}{l}\text { Es la percepción por parte de los interesados, (médicos, } \\
\text { pacientes, personal administrativo, formuladores de políticas } \\
\text { y gestores), de que una intervención es admisible dentro del } \\
\text { sistema que se implementó. Algunos factores relacionados } \\
\text { con la aceptabilidad son la comodidad, la ventaja relativa que } \\
\text { la intervención genera sobre otras intervenciones, la credi- } \\
\text { bilidad de la intervención y de sus autores. La aceptabilidad } \\
\text { incluye aspectos como usabilidad, apariencia, motivación } \\
\text { de uso, desempeño y satisfacción del usuario cuando la } \\
\text { implementación incluye herramientas TIC (Tecnologías de } \\
\text { la información y la comunicación). }\end{array}$ \\
\hline Adopción & $\begin{array}{l}\text { Es el conjunto de intención, decisión y acciones destinadas a } \\
\text { utilizar una nueva intervención. La adopción está relacionada } \\
\text { directamente con la aceptabilidad, dado que refleja la inten- } \\
\text { ción de uso su aprovechamiento y su utilización efectiva, lo } \\
\text { cual sólo se da si la intervención es aceptada. }\end{array}$ \\
\hline Idoneidad & $\begin{array}{l}\text { Es la percepción que tienen los interesados, de que la in- } \\
\text { tervención es pertinente en un entorno, grupo específico y } \\
\text { contexto determinados. La idoneidad refleja la relevancia } \\
\text { de la intervención, su compatibilidad con el contexto de uso } \\
\text { y su utilidad en el entorno natural. }\end{array}$ \\
\hline Viabilidad & $\begin{array}{l}\text { Es la posibilidad que se pueda ejecutar la intervención en un } \\
\text { entorno, grupo específico y contexto determinados. Refleja } \\
\text { la implementación en la práctica real. }\end{array}$ \\
\hline Fidelidad & $\begin{array}{l}\text { Es el grado en que una intervención se ejecute de acuerdo a } \\
\text { su formulación inicial. Refleja la calidad de la ejecución del } \\
\text { programa de implementación y la capacidad del programa } \\
\text { de mantener el objetivo de desplegar en la práctica real las } \\
\text { acciones que tienen respaldo en la evidencia. }\end{array}$ \\
\hline Costo & $\begin{array}{l}\text { Es la cuantía adicional que se debe pagar por la ejecución } \\
\text { de la estrategia. El costo total de la implementación incluye } \\
\text { el costo del proceso de implementación y el costo de la } \\
\text { intervención en sí misma. }\end{array}$ \\
\hline Cobertura & $\begin{array}{l}\text { Es el alcance que tiene la intervención. Proporción de la } \\
\text { población (o del grupo objetivo de la intervención) que } \\
\text { recibe efectivamente la intervención. Involucra el alcance } \\
\text { y acceso a los servicios, es decir, es una característica que } \\
\text { define la cobertura real, teniendo en cuenta las personas } \\
\text { que necesitan la intervención con la calidad esperada, de } \\
\text { tal modo que se combina con la fidelidad para conseguir la } \\
\text { integración en un servicio. }\end{array}$ \\
\hline Sostenibilidad & $\begin{array}{l}\text { Es el grado en que se institucionaliza una intervención o } \\
\text { se mantiene en el tiempo. Implica la durabilidad y mante- } \\
\text { nimiento de la intervención en un entorno de práctica real. }\end{array}$ \\
\hline
\end{tabular}

barreras, actitudes y receptividad hacia el cambio, generados por la implementación de la intervención (17). Las medidas cualitativas incluyen métodos como la entrevista semiestructurada, los grupos focales y la observación en tiempo real de los procesos de atención (27).

Ambas metodologías (cuantitativas y cualitativas) son complementarias, dado que dan cuenta de valoraciones diferentes, alrededor de un mismo fenómeno, por ejemplo, una escala determina el grado o magnitud de la integración de un servicio dentro de una organización de salud, mientras que el grupo focal da cuenta del porqué de la escasa integración del servicio, en la práctica diaria de la misma organización (50).

De otra parte, una valoración sobre la adherencia a una PBE también puede ser valorada a través de métodos cualitativos o cuantitativos, mientras que los datos administrativos pueden ser utilizados cuantitativamente para determinar tasas de utilización de una práctica o en la determinación de cambios en tasas diagnósticas luego del despliegue (51).

Los datos cualitativos pueden a su vez ser analizados a través de teoría fundamentada anclada, es decir, libre de hipótesis previas $(52,53)$ o pueden ser evaluados mediante análisis directo de contenido para determinar la existencia de dimensiones preespecificadas (54).

Las fuentes de datos también incluyen elementos estructurales y ambientales, como pueden ser la estructura física y tecnológica del lugar de implementación, así como de fuentes documentales que incluyen políticas públicas, normas y costos. Datos que en conjunto, sirven para precisar el desenlace o resultado de la implementación (55), el cual se define como el conjunto de efectos que deliberadamente se pretende generar al desplegar tratamientos, practicas o servicios en escenarios reales (56).

El desenlace de la implementación tiene como función servir como un indicador del éxito de la implementación, ser el indicador del proceso de implementación y ser el intermediario entre los desenlaces clínicos y la valoración de la calidad del cuidado (56). Las medidas de desenlace de los estudios de implementación se resumen en la Tabla 2.

\section{Investigación en ciencias de la implementación}

A pesar de las definiciones existentes de los resultados o desenlaces, a través de los cuales se evalúa la implementación, persisten las dificultades para identificar los factores que permiten reconocer el éxito o fracaso del proceso (57). Este aspecto sustenta la necesidad de fortalecer los métodos utilizados por la Ciencia de la Implementación, con el fin de promover el desarrollo de métodos que afinen la evaluación y consoliden la integración del conocimiento en la práctica rutinaria (12).

Se recalca que la implementación en sí, es un proceso, mientras que la Ciencia de la Implementación está orientada a estudiar aquellos aspectos que afectan el despliegue de la intervención, así como los resultados de emplear la imple- 
mentación en entornos reales. Es así como a esta ciencia se le conoce también como la disciplina de la transferencia e integración de conocimiento (58). La investigación en la implementación permite comprender los factores que influyen en los resultados de la implementación, así como sus determinantes durante el proceso, al mismo tiempo que desarrolla su propia metodología y fundamentación teórica $(12,58)$ (Tabla 3$)$.

Se han propuesto tres bases fundamentales sobre las cuales se fundamenta la investigación en implementación. La primera, es el conjunto de marcos o modelos que describen cuál es la mejor estrategia de implementación de acuerdo con el contexto de la práctica. La segunda base implica, el marco explicativo necesario para comprender los resultados de la implementación y la tercera base es el modelo o marco que evalúa la implementación (58). A partir de estas bases se distinguen diversas categorías de modelos y teorías que abarcan los procesos de desarrollo de la implementación.

El desarrollo de modelos que enmarquen el proceso de implementación se ha originado en diversas disciplinas afines a la salud $(12,59)$, por ejemplo, el Modelo de IOWA fue desarrollado por el Departamento de Enfermería de la Universidad de Iowa, el cual aboga porqué el proceso de PBE, debe considerar el conocimiento del sistema de salud en su totalidad (60), incluyendo las particularidades del sistema, de los proveedores y de los pacientes (61).

El modelo de Otawa destaca la naturaleza dinámica de la implementación, centrándose principalmente en los pacientes (62), dado que este modelo considera que el principal objetivo de la PBE son los desenlaces de salud (63). De otra parte existen otros modelos que determinan barreras o facilitadores y se centran en establecer, previo al proceso de implementación, las características del contexto que podrán obstaculizar o favorecer el proceso (64). Por ejemplo el TDF (Theoretical Domains Framework) es un conjunto de 14 dominios que evalúa, sintetiza y explica las conductas de servidores de la salud, relacionadas con barreras y facilita- dores de la asimilación de intervenciones, las cuales tengan por objetivo establecer cambios de comportamiento organizacional (65). De otra parte, el CFIR (The Consolidated Framework for Implementation Research) es un marco que pretende comprender las razones por las cuales un proceso de implementación puede o no ser exitoso, a través de cinco dominios que incluyen la descripción de las características de la intervención, características de los individuos y del propio proceso de implementación $(66,67)$.

Los modelos de procesos y de determinantes requieren de marcos teóricos explicativos que aclaren las razones por las cuales un proceso de implementación puede ser o no exitoso, estos marcos teóricos pueden proceder de ciencias no relacionadas con salud dado que han sido desarrollados a lo largo de la implementación de procesos de innovación en áreas como la administración o las ingenierías y que pueden dar explicaciones a procesos de gran complejidad como la implementación de innovaciones en el sector salud (68). Por ejemplo, la capacidad de una organización para aceptar un cambio (Theory of Organizational Readiness for Change), se entiende como un constructo que describe como los miembros de una organización están dispuestos a implementar un cambio (compromiso con el cambio) y como comparten y desarrollan el sentimiento común de lograrlo (eficacia en el proceso de cambio) (69). Dicho constructo que se origina en la gestión y administración de empresas que buscan cambios de paradigma e implementar estrategias de innovación y desarrollo, concepto que se aplica adecuadamente a los procesos de implantación en salud (70), dado que se trata de determinar, ya sea en una institución hospitalaria o, en un sistema de salud, si los miembros tienen compromiso frente a las innovaciones y si el proceso para adoptar las innovaciones es eficaz, de tal modo que la implementación contiene características que potencian las ventajas del contexto de despliegue de la intervención y plantea soluciones frente a las barreras de asumir las innovaciones, hasta convertirlas en $\operatorname{PBE}(71,72)$.

Tabla 3. Principales modelos y teorías que determinan la metodología empleada por la Ciencia de la Implementación.

\begin{tabular}{|c|c|c|}
\hline Categorías & Definición & Ejemplo \\
\hline Modelos de procesos & $\begin{array}{l}\text { Descripción de modelos que indican el proceso de traslación de } \\
\text { la evidencia a la práctica cotidiana }\end{array}$ & $\begin{array}{l}\text { Modelo IOWA } \\
\text { Modelo Ottawa }\end{array}$ \\
\hline Marcos de determinantes & $\begin{array}{l}\text { Desarrollo de modelos que establecen los factores determinantes } \\
\text { (Barreras o facilitadores) de la implementación }\end{array}$ & $\begin{array}{l}\text { CFIR (The Consolidated Framework for Implementation Research) } \\
\text { TDF (Theoretical Domains Framework) }\end{array}$ \\
\hline Teorías clásicas & $\begin{array}{l}\text { Teorías originadas en ciencias externas a la implementación en } \\
\text { medicina, que contribuyen a explicar y comprender los resultados } \\
\text { y factores determinantes de la implementación }\end{array}$ & $\begin{array}{l}\text { Teoría de las Organizaciones } \\
\text { Teoría de la Difusión de Innovaciones }\end{array}$ \\
\hline Teorías de implementación & $\begin{array}{l}\text { Teorías que surgen a partir de los procesos de investigación en im- } \\
\text { plementación (o se adaptan de teorías previas) y que contribuyen } \\
\text { a explicar y comprender los resultados y factores determinantes } \\
\text { de la implementación. }\end{array}$ & $\begin{array}{l}\text { Modelo COM-B } \\
\text { (Capability Opportunity Motivation Behaviour System) }\end{array}$ \\
\hline Marcos de evaluación & $\begin{array}{l}\text { Modelos específicamente diseñados para evaluar los procesos } \\
\text { de implementación }\end{array}$ & $\begin{array}{l}\text { Modelo PRECEDE-PROCEDE (Predisposing, Reinforcing and } \\
\text { Enabling Constructs in Educational Diagnosis and Evaluation- } \\
\text { Policy, Regulatory, and Organizational Constructs in Educational } \\
\text { and Environmental Development) }\end{array}$ \\
\hline
\end{tabular}


De otra parte la sociología ha propuesto modelos como la Teoría de la Difusión de Innovaciones (Diffusion of Innovations Theory), en el cual se estudian los factores que establecen lo que significa innovación para un individuo, así como las condiciones y canales a través de los cuales se divulga y se adopta la innovación, un modelo adecuado para ser empleado en procesos de implementación en salud, dado que la percepción sobre lo que es innovador o no, depende de la evidencia con que se presente a los interesados (pacientes, médicos), de su entorno cultural y social y de los canales de comunicación que se utilicen para hacer difusión de la innovación, de tal forma que la adopción de una intervención depende del medio y forma en que se difunda dicha novedad (73), que para el caso de la salud, puede ser un programa novedoso o una intervención respaldada por evidencia, pero que requiere convertirse en una PBE (74).

Más allá de uso de modelos originados en ciencias externas, la implementación comienza a originar sus propios modelos a partir de la experiencia recogida en cada proceso (75). Por ejemplo, el COM-B (Capability Opportunity Motivation Behaviour System) es un sistema de cualificación que enlaza las características de la intervención con el análisis de los comportamientos que deben modificarse, para adoptar la intervención, durante el proceso de implementación (76). Finalmente es necesario que el proceso sea evaluado bajo un marco conceptual que sea útil dentro en el diseño y planeación de las intervenciones y que dé cuenta del resultado luego de la implementación $(77,78)$, por ejemplo el modelo PRECEDE-PROCEDE (Predisposing, Reinforcing and Enabling Constructs in Educational Diagnosis and Evaluation-Policy) propone una estrategia para la planeación eficiente de programa de salud, basada en 8 fases que parten desde el contexto social y concluye con la evaluación de desenlaces luego de la implementación (79).

\section{Conclusiones}

La ciencia de la implementación es fundamental para que las intervenciones en salud, respaldadas por evidencia científica, se pongan en práctica, a través de un proceso estructurado y planeado que tenga en cuenta el contexto de los sitos de despliegue. El estudio de implementación recoge diversas características que tiene en común con estudios epidemiológicos clásicos e incluso se puede desarrollarse como un estudio hibrido que comprenda la evaluación de desenlaces clínicos y la evaluación de desenlaces propios de la implementación. La naturaleza particular de la implementación en entornos reales, obliga a que se desarrollen este tipo de estudios a la luz de preguntas específicas de investigación, definiciones precisas de los desenlaces y marcos conceptuales y teóricos que permitan comprender los resultados del proceso. La ciencia de la implementación debe desarrollarse, difundirse y ejecutarse, para que sea posible alcanzar el objetivo de llevar a la práctica cotidiana intervenciones en salud de probada eficacia.

\section{Referencias}

1. Habets, M.G., J.J. van Delden, and A.L. Bredenoord, The social value of clinical research. BMC Med Ethics, 2014. 15: p. 66.

2. Higgins, A.M. and A.H. Harris, Health economic methods: cost-minimization, cost-effectiveness, cost-utility, and cost-benefit evaluations. Crit Care Clin, 2012. 28(1): p. 11-24, v.

3. Sessler, D.I. and P.B. Imrey, Clinical Research Methodology 1: Study Designs and Methodologic Sources of Error. Anesth Analg, 2015. 121(4): p. 1034-42.

4. Yang, L.J., K.W. Chang, and K.C. Chung, Methodologically rigorous clinical research. Plast Reconstr Surg, 2012. 129(6): p. 979e-988e.

5. Thiese, M.S., Observational and interventional study design types; an overview. Biochem Med (Zagreb), 2014. 24(2): p. 199-210.

6. Murad, M.H., Clinical Practice Guidelines: A Primer on Development and Dissemination. Mayo Clin Proc, 2017. 92(3): p. 423-433.

7. Sur, R.L. and P. Dahm, History of evidence-based medicine. Indian J Urol, 2011. 27(4): p. 487-9.

8. Charles, C., A. Gafni, and E. Freeman, The evidence-based medicine model of clinical practice: scientific teaching or belief-based preaching? J Eval Clin Pract, 2011. 17(4): p. 597-605.

9. Colditz, G.A., et al., Translating science to practice: community and academic perspectives. J Public Health Manag Pract, 2008. 14(2): p. 144-9.

10. Nilsen, P., et al., Never the twain shall meet?--a comparison of implementation science and policy implementation research. Implementation science : IS, 2013. 8: p. 63.

11. Smits, P.A. and J.L. Denis, How research funding agencies support science integration into policy and practice: an international overview. Implementation science : IS, 2014. 9: p. 28.

12.Rumbo, J., et al., Implementar evidencias e investigar en implementación: dos realidades diferentes y prioritarias. Enfermería Clínica, 2016. 26(2): p. 382-386.

13. Homer-Vanniasinkam, S. and J. Tsui, The continuing challenges of translational research: clinician-scientists' perspective. Cardiol Res Pract, 2012. 2012: p. 246710 .

14. Eccles, M.P. and B.S. Mittman, Welcome to Implementation Science. Implementation Science, 2006. 1(1): p. 1

15. Sturke, R., et al., A multi-disciplinary approach to implementation science: the NIH-PEPFAR PMTCT implementation science alliance. Journal of acquired immune deficiency syndromes, 2014. 67 Suppl 2: p. S163-7.

16. Proctor, E.K., et al., Writing implementation research grant proposals: ten key ingredients. Implementation science : IS, 2012. 7: p. 96.

17. Bauer, M.S., et al., An introduction to implementation science for the nonspecialist. BMC Psychol, 2015. 3: p. 32.

18. Morrison, D., Clinical inference: critically weighing the evidence from trials and registries to make clinical decisions. Catheter Cardiovasc Interv, 2008. 72(3): p. 381-5.

19. Steckler, A. and K.R. McLeroy, The importance of external validity. Am J Public Health, 2008. 98(1): p. 9-10.

20. Tunis, S.R., D.B. Stryer, and C.M. Clancy, Practical clinical trials: increasing the value of clinical research for decision making in clinical and health policy. JAMA, 2003. 290(12): p. 1624-32.

21.Curran, G.M., et al., Effectiveness-implementation hybrid designs: combining elements of clinical effectiveness and implementation research to enhance public health impact. Med Care, 2012. 50(3): p. 217-26.

22. Pfadenhauer, L.M., et al., Making sense of complexity in context and implementation: the Context and Implementation of Complex Interventions (CICI) framework. Implement Sci, 2017. 12(1): p. 21.

23. Powell, B.J., et al., A refined compilation of implementation strategies: results from the Expert Recommendations for Implementing Change (ERIC) project. Implementation science : IS, 2015. 10: p. 21.

24. Shidhaye, R., Implementation Science for closing the treatment gap for mental disorders by translating evidence base into practice: experiences from the PRIME project. Australasian psychiatry : bulletin of Royal Australian and New Zealand College of Psychiatrists, 2015. 23(6 Suppl): p. 35-7.

25. Kindig, D. and G. Stoddart, What Is Population Health? American Journal of Public Health, 2003. 93(3): p. 380-383.

26. Jackson, C.L., et al., Implementation research - its importance and application in primary care. Med J Aust, 2014. 201(3 Suppl): p. S42-3.

27. Tumilowicz, A., L.M. Neufeld, and G.H. Pelto, Using ethnography in implementation research to improve nutrition interventions in populations. Matern Child Nutr, 2015. 11 Suppl 3: p. 55-72.

28. Meissner, H.I., et al., The U.S. training institute for dissemination and implementation research in health. Implementation science : IS, 2013. 8: p. 12. 
29. Jacobs, S.R., B.J. Weiner, and A.C. Bunger, Context matters: measuring implementation climate among individuals and groups. Implementation science : IS, 2014. 9: p. 46.

30. Margolis, P., et al., Quality improvement, clinical research, and quality improvement research--opportunities for integration. Pediatr Clin North Am, 2009. 56(4): p. 831-41.

31. Tumilowicz, A., et al., Using implementation research for evidence-based programme development: a case study from Kenya. Matern Child Nutr, 2015. 11 Suppl 3: p. 1-5.

32. Song, F., et al., Dissemination and publication of research findings: an updated review of related biases. Health Technol Assess, 2010. 14(8): p. iii, ix-xi, 1-193.

33. Langlois, E.V., et al., Enhancing evidence informed policymaking in complex health systems: lessons from multi-site collaborative approaches. Health Res Policy Syst, 2016. 14: p. 20.

34. Suárez-Obando, F., et al., Portal de Guías de Práctica Clínica: estrategia digital (e-learning) para la difusión de guías de práctica clínica desarrolladas en Colombia. Universitas Medica, 2016. 57(1): p. 44-57.

35. De Angelis, G., et al., Information and Communication Technologies for the Dissemination of Clinical Practice Guidelines to Health Professionals: A Systematic Review. JMIR Med Educ, 2016. 2(2): p. e16.

36. Ray-Coquard, I., et al., A controlled "before-after" study: impact of a clinical guidelines programme and regional cancer network organization on medical practice. Br J Cancer, 2002. 86(3): p. 313-21.

37. Duarte, A., A. Torres, and V.C., Manual de implementación de guías de práctica clínica basadas en evidencia, en instituciones prestadoras de servicios de salud en Colombia. 2014, Bogotá D.C., Colombia: Ministerio de Salud y Protección Social.

38. Hurst, D. and S. Mickan, Describing knowledge encounters in healthcare: a mixed studies systematic review and development of a classification. Implement Sci, 2017. 12(1): p. 35.

39. Clemson, L., et al., Integrated solutions for sustainable fall prevention in primary care, the iSOLVE project: a type 2 hybrid effectiveness-implementation design. Implement Sci, 2017. 12(1): p. 12.

40. Williams, D., et al., Engagement of the medical-technology sector with society. Sci Transl Med, 2017.9(385).

41. Lobb, R. and G.A. Colditz, Implementation Science and its Application to Population Health. Annual review of public health, 2013. 34: p. 235-251.

42. Jager, C., et al., A tailored programme to implement recommendations for multimorbid patients with polypharmacy in primary care practices-process evaluation of a cluster randomized trial. Implement Sci, 2017. 12(1): p. 31.

43. Peters, D., N. Tran, and T. Adam, ¿Qué es la Investigación sobre la implementaciónde políticas?, in Investigación sobre la implementación de políticas de salud: Guía práctica. 2014, Organización Mundial de la Salud: Ginebra, Suiza. .

44. Wierenga, D., et al., What is actually measured in process evaluations for worksite health promotion programs: a systematic review. BMC Public Health, 2013. 13: p. 1190.

45. Hoekstra, F., et al., Design of a process evaluation of the implementation of a physical activity and sports stimulation programme in Dutch rehabilitation setting: ReSpAct. Implement Sci, 2014. 9: p. 127.

46. Stetler, C.B., et al., The role of formative evaluation in implementation research and the QUERI experience. J Gen Intern Med, 2006. 21 Suppl 2: p. S1-8.

47. Radjaeipour, G., D.W. Chambers, and M. Geissberger, Can performance on summative evaluation of wax-added dental anatomy projects be better predicted from the combination of supervised and unsupervised practice than from supervised practice alone? Eur J Dent Educ, 2016. 20(4): p. 237-240.

48. Ament, S.M., et al., A detailed report of the resource use and costs associated with implementation of a short stay programme for breast cancer surgery. Implement Sci, 2015. 10: p. 78.

49. Unertl, K.M., et al., Traversing the many paths of workflow research: developing a conceptual framework of workflow terminology through a systematic literature review. J Am Med Inform Assoc, 2010. 17(3): p. 265-73.

50. Padwa, H., et al., The Implementation of Integrated Behavioral Health Protocols In Primary Care Settings in Project Care. J Subst Abuse Treat, 2016. 62: p. 74-83.

51. Carey, M., et al., Improving adherence to colorectal cancer surveillance guidelines: results of a randomised controlled trial. BMC Cancer, 2017. 17(1): p. 106

52. Madsen, W.C., Narrative Approaches to Organizational Development: A Case Study of Implementation of Collaborative Helping. Fam Process, 2016. 55(2): p. 253-69.

53. Hallum-Montes, R., et al., Barriers and Facilitators to Health Center Implemen- tation of Evidence-Based Clinical Practices in Adolescent Reproductive Health Services. J Adolesc Health, 2016. 58(3): p. 276-83.

54. Kazak, A.E., et al., Provider Perspectives on the Implementation of Psychosocial Risk Screening in Pediatric Cancer. J Pediatr Psychol, 2017.

55. Cheng, H.G. and M.R. Phillips, Secondary analysis of existing data: opportunities and implementation. Shanghai Arch Psychiatry, 2014. 26(6): p. 371-5.

56. Proctor, E., et al., Outcomes for implementation research: conceptual distinctions, measurement challenges, and research agenda. Adm Policy Ment Health, 2011. 38(2): p. 65-76.

57. Hodge, L.M. and K.M. Turner, Sustained Implementation of Evidence-based Programs in Disadvantaged Communities: A Conceptual Framework of Supporting Factors. Am J Community Psychol, 2016. 58(1-2): p. 192-210.

58. Nilsen, P., Making sense of implementation theories, models and frameworks. Implement Sci, 2015. 10: p. 53.

59. Cook, J.M., et al., Measurement of a model of implementation for health care: toward a testable theory. Implement Sci, 2012. 7: p. 59.

60. Doody, C.M. and O. Doody, Introducing evidence into nursing practice: using the IOWA model. Br J Nurs, 2011. 20(11): p. 661-4.

61. Newhouse, R., et al., Evidence-based practice: a practical approach to implementation. J Nurs Adm, 2005. 35(1): p. 35-40.

62. Manojlovich, M., et al., Hiding in plain sight: communication theory in implementation science. Implement Sci, 2015. 10: p. 58.

63. National Collaborating Centre for Methods and Tools (2010). Ottawa Model of Research Use: A Framework for Adopting Innovations. 2010 (cited 2017 April 15); Available from: http://www.nccmt.ca/resources/search/65.

64. Birken, S.A., et al., Combined use of the Consolidated Framework for Implementation Research (CFIR) and the Theoretical Domains Framework (TDF): a systematic review. Implement Sci, 2017. 12(1): p. 2.

65. Mosavianpour, M., et al., Theoretical domains framework to assess barriers to change for planning health care quality interventions: a systematic literature review. J Multidiscip Healthc, 2016. 9: p. 303-10.

66. Kirk, M.A., et al., A systematic review of the use of the Consolidated Framework for Implementation Research. Implement Sci, 2016. 11: p. 72.

67. Breimaier, H.E., et al., The Consolidated Framework for Implementation Research (CFIR): a useful theoretical framework for guiding and evaluating a guideline implementation process in a hospital-based nursing practice. BMC Nurs, 2015. 14: p. 43.

68. Shortell, S.M., Applying Organization Theory to Understanding the Adoption and Implementation of Accountable Care Organizations. Medical Care Research and Review, 2016. 73(6): p. 694-702.

69. Weiner, B.J., A theory of organizational readiness for change. Implementation science : IS, 2009. 4: p. 67.

70. Sanders, K.A., et al., Organizational readiness for change: Preceptor perceptions regarding early immersion of student pharmacists in health-system practice. Res Social Adm Pharm, 2017

71. Helfrich, C.D., et al., Predicting implementation from organizational readiness for change: a study protocol. Implementation science : IS, 2011. 6: p. 76.

72. Benzer, J.K., et al., The role of organizational structure in readiness for change: A conceptual integration. Health services management research, 2017. 30(1): p. 34-46.

73. Olsson, A., K. Skovdahl, and M. Engstrom, Using diffusion of innovation theory to describe perceptions of a passive positioning alarm among persons with mild dementia: a repeated interview study. BMC Geriatr, 2016. 16: p. 3.

74. McMullen, H., et al., Explaining high and low performers in complex intervention trials: a new model based on diffusion of innovations theory. Trials, 2015. 16: p. 242 .

75. Rapport, F., et al., The struggle of translating science into action: Foundational concepts of implementation science. J Eval Clin Pract, 2017.

76. Michie, S., M.M. van Stralen, and R. West, The behaviour change wheel: a new method for characterising and designing behaviour change interventions. Implement Sci, 2011. 6: p. 42.

77. Milat, A.J. and B. Li, Narrative review of frameworks for translating research evidence into policy and practice. Public Health Res Pract, 2017. 27(1).

78. Donaldson, N.E., D.N. Rutledge, and J. Ashley, Outcomes of adoption: measuring evidence uptake by individuals and organizations. Worldviews Evid Based Nurs, 2004. 1 Suppl 1: p. S41-51.

79. Ghaffarifar, S., et al., Barriers to Effective Doctor-Patient Relationship Based on PRECEDE PROCEED Model. Glob J Health Sci, 2015. 7(6): p. 24-32. 